\title{
Design and high order optimization of the Accelerator Test Facility lattices
}

\author{
E. Marin, ${ }^{1,2, *}$ R. Tomás, ${ }^{2}$ P. Bambade,${ }^{3}$ K. Kubo, ${ }^{4}$ T. Okugi, ${ }^{4}$ T. Tauchi ${ }^{4}$ N. Terunuma, ${ }^{4}$ \\ J. Urakawa, ${ }^{4}$ A. Seryi, ${ }^{5}$ G. R. White, ${ }^{1}$ and M. Woodley ${ }^{1}$ \\ ${ }^{1}$ SLAC National Accelerator Laboratory, 2575 Sand Hill Road, Menlo Park, California 94025-7015, USA \\ ${ }^{2}$ European Organization for Nuclear Research (CERN), CH-1211 Geneva 23, Switzerland \\ ${ }^{3}$ LAL, Universite Paris-Sud, CNRS/IN2P3, Orsay, France \\ ${ }^{4}$ High Energy Accelerator Research Organization (KEK), 1-1 Oho, Tsukuba, Ibaraki 305-0801, Japan \\ ${ }^{5}$ John Adams Institute for Accelerator Science at University of Oxford, Denys Wilkinson Building, \\ Keble Road, Oxford OX1 3RH, United Kingdom
}

(Received 11 July 2013; published 12 February 2014)

\begin{abstract}
The Accelerator Test Facility 2 (ATF2) aims to test the novel chromaticity correction scheme which is implemented in the final focus systems of future linear colliders such as the International Linear Collider (ILC) and the Compact Linear Collider (CLIC). The ATF2 nominal and ultralow $\beta^{*}$ lattices are designed to vertically focus the beam at the focal point, or usually referred to as interaction point (IP), down to 37 and $23 \mathrm{~nm}$, respectively. The vertical chromaticities of the nominal and ultralow $\beta^{*}$ lattices are comparable to those of ILC and CLIC, respectively. When the measured multipole components of the ATF2 magnets are considered in the simulations, the evaluated spot sizes at the IP are well above the design values. In this paper we describe the analysis of the high order aberrations that allows identifying the sources of the observed beam size growth. In order to recover the design spot sizes three solutions are considered, namely final doublet replacement, octupole insertion, and optics modification. Concerning the future linear collider projects, the consequences of magnetic field errors of the focusing quadrupole magnet of the final doublet are also addressed.
\end{abstract}

DOI: 10.1103/PhysRevSTAB.17.021002

PACS numbers: 29.27.Eg, 41.85.-p, 41.85.Gy, 29.20.Ej

\section{INTRODUCTION}

The accelerator and particle physics communities are considering a lepton linear collider as the most appropriate machine to carry out high precision particle physics research in the high energy regime. There exist two proposals for the next generation of $\mathrm{e}^{+}-\mathrm{e}^{-}$linear collider (LC), the International Linear Collider (ILC) [1-3] and the Compact Linear Collider (CLIC) [4-6]. In order to reach the required luminosity $(\mathcal{L})$ for the experiments, the vertical spot size at the interaction point (IP) $\left(\sigma_{y}^{*}\right)$ is of the order of a few nanometers. At the nm beam size regime the energy spread of the incoming beam leads to a noticeable beam size growth mainly due to the chromaticity $\xi$ generated by the final doublet (FD) quadrupole magnets. The final focus systems (FFS) of both projects of $\mathrm{e}^{+}-\mathrm{e}^{-}$LCs are based on the local chromaticity correction scheme proposed in [7] which has recently been validated experimentally [8] at the Accelerator Test Facility (ATF2); see [9-11] for a detailed description.

"emarinla@slac.stanford.edu

Published by the American Physical Society under the terms of the Creative Commons Attribution 3.0 License. Further distribution of this work must maintain attribution to the author(s) and the published article's title, journal citation, and DOI.
The ATF2 nominal lattice is a scaled-down version of the ILC FFS, which features a value of $\sigma_{y}^{*}=37 \mathrm{~nm}^{1}$ if an error-free lattice is considered in the simulation. The ATF2 ultralow $\beta^{*}$ lattice [12] is a proposal for an even more challenging optics with a value of vertical beta function at the IP $\left(\beta_{y}^{*}\right)$ a quarter of that of the ATF2 nominal lattice. The expected $\sigma_{y}^{*}$ is equal to $23 \mathrm{~nm}^{1}$ if an error-free lattice is considered. The goal of the ATF2 ultralow $\beta^{*}$ lattice is to test the feasibility of a lattice with a chromaticity comparable to that of the CLIC FFS. Table I shows the relevant parameters of the ATF2, CLIC, and ILC designs, with special attention to the vertical chromaticity, shown in the last column, estimated as $\approx \frac{L^{*}}{\beta_{v}^{*}}$. As can be seen, the calculated value of the chromaticity of the ATF2 ultralow $\beta^{*}$ lattice is comparable to the one of CLIC and almost a factor 4 larger than the one of the ATF2 nominal lattice. The parameters of the final focus test beam (FFTB) $[13,14]$ are also included in Table I for comparison purposes. This accelerator was a prototype of a final focus system based on a nonlocal chromaticity correction scheme. In 1994 a vertical spot size of about $70 \mathrm{~nm}$ [15] was achieved at the focal point of the FFTB. At ATF2 it has been recently reached and further reduced the minimum $\sigma_{y}^{*}$ measured at FFTB; see [8].

\footnotetext{
${ }^{1} \mathrm{~A}$ relative energy spread $(\Delta p / p)$ of $0.08 \%$ and high order aberrations of the beam are considered in the beam size calculation.
} 
TABLE I. Comparison between relevant parameters of different final focus systems.

\begin{tabular}{llcccccc}
\hline \hline Project & Status & Beam energy $[\mathrm{GeV}]$ & $\gamma \epsilon_{y}[\mathrm{~nm}]$ & $\sigma_{y}^{*}[\mathrm{~nm}]$ & $\beta_{y}^{*}[\mathrm{~mm}]$ & $\mathrm{L}^{*}[\mathrm{~m}]$ & $\xi_{y}$ \\
\hline FFTB & Designed & 46.6 & 2000 & 52 & 0.1 & 0.4 \\
FFTB & Measured & 46.6 & 2000 & 70 & $\ldots$ & 0.4 \\
ATF2 nominal & Designed & 1.3 & 30 & 37 & 0.1 & 1.0 \\
ATF2 nominal & Measured & 1.3 & 30 & $65^{\text {a }}$ & 0.1 & 1.0 \\
ATF2 ultralow $\beta^{*}$ & Proposed & 1.3 & 30 & 23 & 0.025 & 1.0 & 40000 \\
CLIC L* $=3.5 \mathrm{~m}$ & Designed & 1500 & 20 & 1 & 0.069 & 3.5 & 500000 \\
ILC & Designed & 250 & 35 & 5.9 & 0.48 & 3.5 & 7500 \\
\hline \hline
\end{tabular}

${ }^{\mathrm{a}}$ This value is considered as an upper limit of the actual beam size due to relative phase jitter between the laser fringe pattern and the $e^{-}$beam; see more details in [16].

\section{DESCRIPTION OF ATF2}

The ATF2 beam line extends over about $90 \mathrm{~m}$ from the beam extraction point in the ATF damping ring to the IP. Figure 1 shows a layout of the ATF2 beam line.

The ATF2 beam line is divided into two sections, the extraction beam line (EXT) and the final focus system. The EXT extends over $52 \mathrm{~m}$; it comprises an extraction and a diagnostics section. The diagnostics section is used for measuring the emittance and the Twiss parameters and for correcting the dispersion and transverse coupling of the electron beam. The ATF2 FFS beam line extends over $40 \mathrm{~m}$; it is responsible for transporting and vertically focusing the beam at the IP to tens of nanometers. It consists of a matching section composed of six quadrupole magnets (denoted by QM16FF, QM15FF, QM14FF, QM13FF, QM12FF, and QM11FF) whose function is to match the $\beta$ functions measured in the EXT diagnostics section. In addition there are 14 quadrupole magnets which transport the beam to the FD that include one focusing
(QF1FF) and one defocusing (QD0FF) quadrupoles meant to focus the transverse beam size at the IP. The last nine quadrupoles of the EXT beam line plus the transport quadrupoles of the FFS are referred to as QEA magnets. Three bending magnets, namely B1FF, B2FF, and B5FF, generate the required dispersion to correct the chromaticity by means of the five normal sextupoles, namely SF6FF, SF5FF, SD4FF, SF1FF, and SD0FF. In addition four skew sextupoles, namely SK1FF, SK2FF, SK3FF, and SK4FF, have been recently installed [17] in the FFS. Figure 2 shows the $\beta_{x, y}$ and $\eta_{x}$ functions along the FFS beam line.

The Shintake monitor [18] installed at ATF2 provides the capability for measuring the small vertical beam size at the IP; its design is described in [19]. Its operating principle is based on the detection of Compton scattered photons coming from the interaction between the electron beam and the interference fringe pattern [20] created by crossing two laser beams. $\sigma_{y}^{*}$ is inferred from the modulation of the measured Compton signal when scanning the interference pattern across the beam. The expected IP vertical spot size

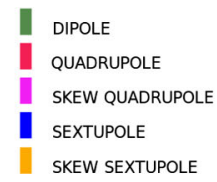

EXTRACTION LINE
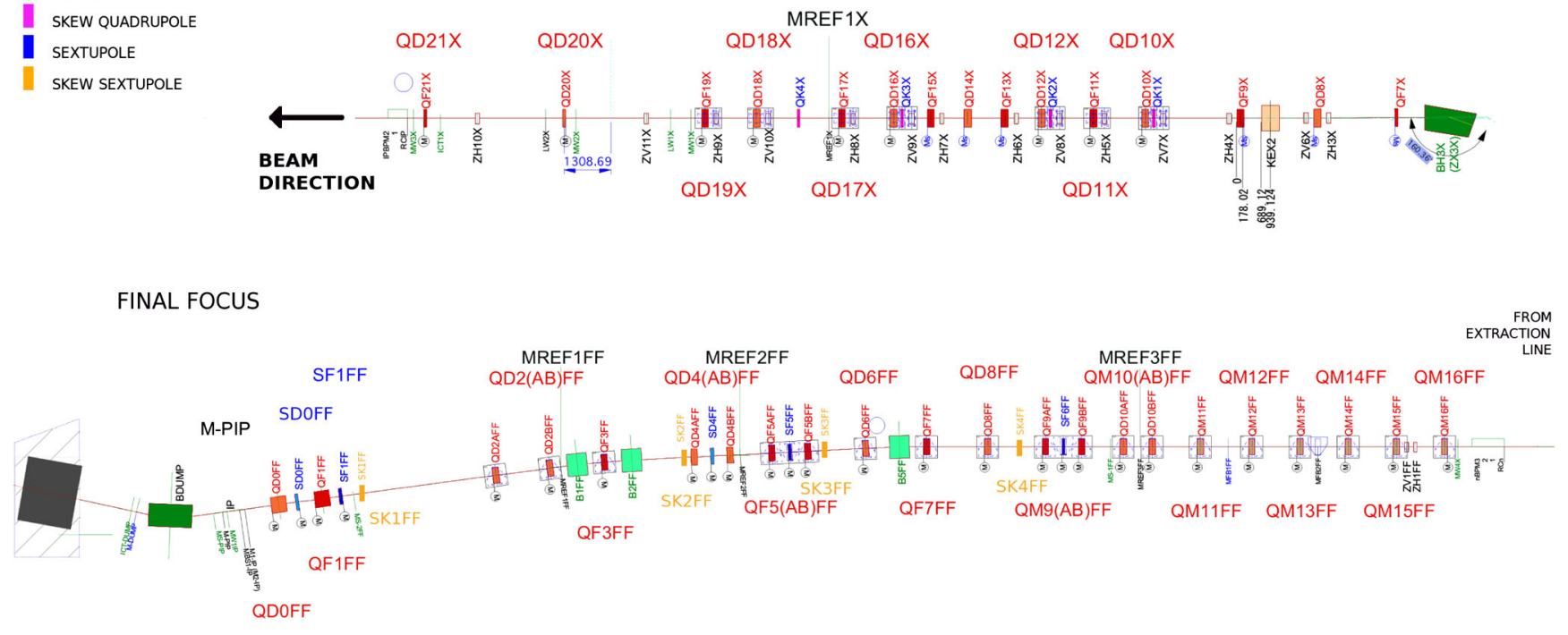

FIG. 1 (color online). Scheme of the ATF2. The beam line on the left represents the extraction beam line (EXT). The beam line on the right represents the FFS as the continuation of the EXT line (the figure is courtesy of Araki). 


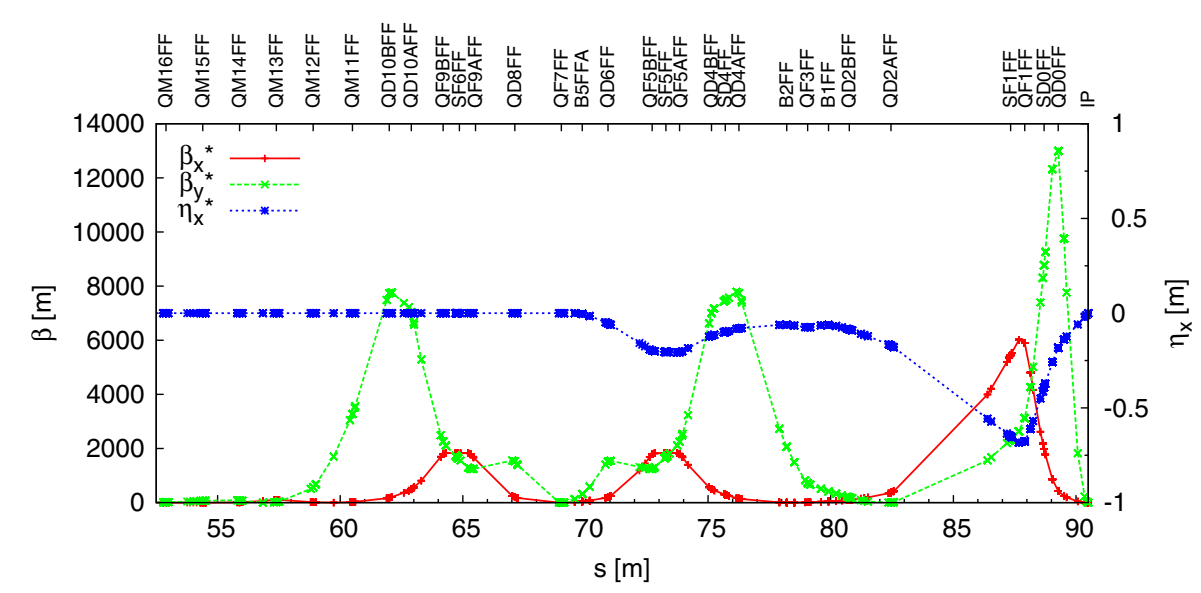

FIG. 2 (color online). The $\beta_{x, y}$ functions and the $\eta_{x}$ function for the ATF2 nominal lattice throughout the ATF2 final focus line.

by the Shintake monitor $\left(\sigma_{y, \text { Shi }}^{*}\right)$ is obtained by a code that simulates the laser-beam interaction, as described in [21].

The values of $\sigma_{y}^{*}$ presented in Table I for the ATF2 and LC projects are evaluated assuming error-free lattices. However, the field quality of the magnets might preclude to reach small beam sizes at the IP. In order to address this issue, the multipole components of the ATF2 FFS magnets have been measured. The impact of these measured multipole components on the IP beam sizes for the ATF2 lattices is presented in this paper. The analysis capabilities of the MAPCLASS code $[22,23]$ are extensively used to identify the multipole components that have a noticeable impact on $\sigma^{*}$ and to propose effective solutions that preserve the design spot sizes at the IP.

\section{IMPACT OF THE MULTIPOLE COMPONENTS OF THE ATF2 MAGNETS}

Data on the multipole components of the ATF2 magnets can be found in [24]. This data was obtained after a careful cross-check between two different magnet measurement campaigns conducted at IHEP and KEK [25]; see [26].

The strength of each measured multipole component is introduced into the MAD-X [27] model of the ATF2 lattices by fixing its ratio relative to the strength of the magnet, so that a variation of the magnet strength modifies the strength of its multipole components linearly. Each multipole component is modeled by thin elements at the edges and at the center of the magnets. The sum of the thin element strengths is equal to the measured integrated strength. With all the multipole components included into the model, the beam size at the IP is found to be larger than expected for both the ATF2 nominal and ultralow $\beta^{*}$ lattices. Three different beam size definitions, core, Shintake, and rms, are used in this study.

The core beam size $\left(\sigma_{\text {core }}\right)$ is the width of a Gaussian distribution fitted to the histogram of a bunch of particles. It is the part of the beam that largely contributes to the luminosity, therefore it is of special interest when referring to linear colliders. By Shintake beam size $\left(\sigma_{\text {Shi }}\right)$ we refer to the value obtained from the convolution between the bunch of particles and the interference pattern of the laser; for more details refer to [20]. The systematic errors from the Shintake monitor are not taken into account in this calculation. The obtained error in the computation due to the finite number of particles (10000) used in the calculations is less than $1 \%$ as explained in [21]. By $r m s^{2}$ beam size $\left(\sigma_{\text {rms }}\right)$ we refer to the following calculation:

$$
\sigma_{\mathrm{rms}}=\sqrt{\int_{-\infty}^{\infty}(u-\bar{u})^{2} \rho d \nu}
$$

where $u$ stands for $x, p_{x}, y, p_{y}$ and $\rho$ is the particle density distribution. The introduced beam sizes are usually ordered as $\sigma_{\text {rms }} \geq \sigma_{\text {Shi }} \geq \sigma_{\text {core }}$ because the contribution from the tails of the bunch to $\sigma$ given by the interference pattern is greater than the one given by the core definition, but lower than the one given by the rms definition. However, when the beam can be represented by a Gaussian distribution, the three definitions coincide.

Depending on the considered beam size definition, the $\Delta \sigma_{y}^{*}$ calculated when the measured multipole components are included in the model ranges from a few to hundreds of percent. Table II summarizes the evaluated spot sizes at the IP when gradually including the measured multipole components. Four different scenarios have been considered according to the multipole component measurements included into the ATF2 model: (i) without multipole components; (ii) only the QEA multipole components; (iii) only the FD multipole components; and (iv) all the multipole components.

For the ATF2 ultralow $\beta^{*}$ lattice the situation is much worse than for the nominal one. The comparison between the $\Delta \sigma_{y}^{*} / \sigma_{y_{0}}^{*}$ of the different beam size definitions suggests

\footnotetext{
${ }^{2}$ The beam sizes presented in this paper refer to the rms definition if not stated otherwise.
} 
TABLE II. Comparison between the values of the IP beam size obtained, when gradually including the multipole components of the ATF2 magnets, according to the core, Shintake, and rms beam size definitions for the ATF2 lattices. The values shown inside the parentheses refer to the percentage of beam size growth with respect to the beam size for the case where no multipoles are assumed in the simulations.

\begin{tabular}{|c|c|c|c|c|}
\hline & \multicolumn{4}{|c|}{ ATF2 nominal lattice } \\
\hline & \multicolumn{2}{|l|}{$\sigma_{x}^{*}[\mu \mathrm{m}]$} & \multicolumn{2}{|l|}{$\sigma_{y}^{*}[\mathrm{~nm}]$} \\
\hline & RMS & CORE & SHINTAKE & RMS \\
\hline No mults & 3.0 & 37.2 & 37.3 & 38.0 \\
\hline All QEA mults & $3.5(+17 \%)$ & $37.3(+0.3 \%)$ & $38.8(+4 \%)$ & $43.2(+14 \%)$ \\
\hline Only FD mults & $3.9(+30 \%)$ & $39.3(+5.6 \%)$ & $41.8(+12 \%)$ & $66.9(+76 \%)$ \\
\hline All mults & $3.9(+30 \%)$ & $39.3(+5.6 \%)$ & $41.8(+12 \%)$ & $66.9(+76 \%)$ \\
\hline \multicolumn{5}{|c|}{ ATF2 ultralow $\beta^{*}$ lattice } \\
\hline No mults & 3.0 & 20.4 & 22.8 & 23.1 \\
\hline All QEA mults & $3.4(+13 \%)$ & $22.6(+11 \%)$ & $26.3(+15 \%)$ & $29.5(+28 \%)$ \\
\hline Only FD mults & $3.7(+23 \%)$ & $30.0(+47 \%)$ & $42.3(+86 \%)$ & $80.1(+247 \%)$ \\
\hline All mults & $3.7(+23 \%)$ & $30.0(+47 \%)$ & $42.3(+86 \%)$ & $80.1(+247 \%)$ \\
\hline
\end{tabular}

that the tails of the bunch are enhanced by the multipole components of the magnets. From Table II it is inferred that the most important sources of beam size increase are the multipole errors of the FD. Figure 3 shows the results of the MAPCLASS analysis for the ATF2 ultralow $\beta^{*}$ lattice. Each curve refers to $\sigma_{y}^{*}$ for different orders. It can be seen that the sextupole (second order) component in green and the dodecapole (fifth order) component in blue, are the main sources of the observed vertical beam size increase. The dependence of $\sigma_{y}^{*}$ on $\gamma \epsilon_{x}$ at the fifth order reveals the presence of a skew dodecapole component.

\section{A. Final doublet replacement}

The final doublet is the major source of vertical beam size increase at the IP, as shown in Table II. Therefore the replacement of the present FD by a better field quality magnet that satisfies the ATF2 nominal and ultralow $\beta^{*}$ tolerances may represent a step forward for the ATF2 facility. The tolerances for the ATF2 nominal and ultralow $\beta^{*}$ lattices are evaluated by MAPCLASS in order to be compared with the multipole components measurements.

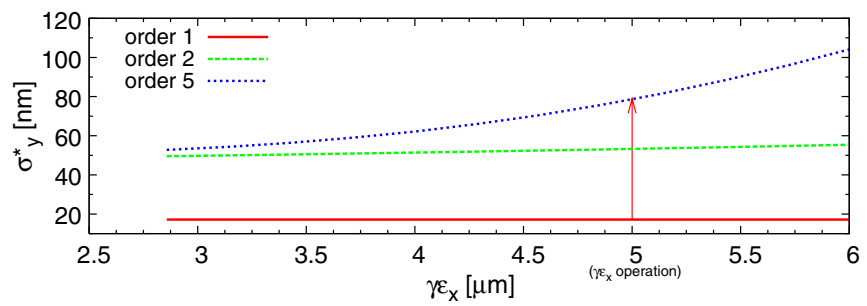

FIG. 3 (color online). Plot of $\sigma_{y}^{*}$ as a function of $\gamma \epsilon_{x}$ for the ATF2 ultralow $\beta^{*}$ lattice. The red curve represents the vertical IP beam size considering up to the quadrupole components, the green dashed curve includes the sextupole components, and the blue dot curve takes into account all of the multipoles up to the dodecapole components.
The sextupole, octupole, decapole, and dodecapole tolerances of QF1FF and QD0FF are determined assuming an error-free ATF2 lattice. The method for the evaluation follows: the value of the normal and skew multipole component is increased one by one until $\sigma_{y}^{*}$ increases by $2 \%$. The columns labeled as Tol.NL and Tol.UL of Table III summarize the obtained tolerances for the ATF2 nominal and ultralow $\beta^{*}$ lattices, respectively. It is observed that tighter tolerances are obtained for the ATF2 ultralow $\beta^{*}$ than for the nominal lattice. In addition tighter skew tolerances than the normal ones are observed for all the multipole components of QF1FF due to the higher value of $\beta_{x}$ at QF1FF. Table III compares the evaluated tolerances of QF1FF and QD0FF for the ATF2 ultralow $\beta^{*}$ lattice with the measured multipole components. The cases for which the magnetic measurement exceeds the tolerance are marked in bold. It also shows that QF1FF exceeds the tolerances in almost all normal and skew multipole components, whereas for QD0FF only the sextupole component exceeds the tolerance. From the analysis of Fig. 3 and the comparison made in Table III, it can be concluded that in order to achieve the design IP spot size for both ATF2 lattices a better field quality QF1FF should be used, as considered in [28]. To this end, in November of 2012 the QF1FF magnet was replaced by a 4Q17 type quadrupole [29] recycled from the PEPII LER accelerator [30]. The 4Q17 magnet has a better field quality with respect to the previous QF1FF as shown in Table IV.

The evaluated $\sigma_{y}^{*}$ for the ATF2 nominal lattice when considering the multipole components of the 4Q17 magnet, after optimizing the sextupole magnets, is $37 \mathrm{~nm}$ which represents a satisfactory solution for this lattice. Concerning the ATF2 ultralow $\beta^{*}$ lattice a $\sigma_{y}^{*}=31 \mathrm{~nm}$ is obtained when assuming the $4 \mathrm{Q} 17$ multipole components, although it represents an important beam size reduction it is not sufficient. The contribution to the 
TABLE III. Comparison between the tolerances evaluated for the ATF2 nominal (Tol.NL) and ultralow $\beta^{*}$ (Tol.UL) lattices and the measured multipole components of QF1FF and QD0FF at a radius (R) equal to $1 \mathrm{~cm}$. Each tolerance represents a $\Delta \sigma_{y}^{*}=2 \%$. In bold letters the cases for which the measured multipole component does not meet the evaluated tolerances for both ATF2 lattices and in italic style when the measurements only meet the tolerance for the ATF2 nominal lattices.

\begin{tabular}{|c|c|c|c|c|c|c|}
\hline \multirow[b]{2}{*}{ Component } & \multicolumn{3}{|c|}{ Normal } & \multicolumn{3}{|c|}{ Skew } \\
\hline & Tol.UL & Measured & Tol.NL & Tol.UL & Measured & Tol.NL \\
\hline & \multicolumn{6}{|c|}{ Sextupole $\left[10^{-4}\right]$} \\
\hline QF1FF & 0.37 & 2.7 & 1.2 & 0.07 & 0.28 & 0.09 \\
\hline QD0FF & 0.2 & 1.84 & 0.8 & 0.18 & 1.76 & 0.4 \\
\hline \multicolumn{7}{|c|}{ Octupole $\left[10^{-4}\right]$} \\
\hline QF1FF & 0.15 & 0.57 & 0.7 & 0.07 & 0.04 & 0.1 \\
\hline QD0FF & 1.8 & 0.44 & 6.0 & 0.8 & 0.28 & 1.2 \\
\hline \multicolumn{7}{|c|}{ Decapole $\left[10^{-4}\right]$} \\
\hline QF1FF & 0.43 & 1.2 & 0.9 & 0.08 & 0.19 & 0.11 \\
\hline QD0FF & 3.15 & 0.65 & 10.1 & 2.9 & 0.32 & 4.3 \\
\hline \multicolumn{7}{|c|}{ Dodecapole $\left[10^{-4}\right]$} \\
\hline QF1FF & 0.17 & 3.4 & 0.4 & 0.09 & 0.76 & 0.11 \\
\hline QD0FF & 15.0 & 3.5 & 50.0 & 9.0 & 0.22 & 12.3 \\
\hline
\end{tabular}

remaining beam size growth is mainly due to the QEA quadrupole magnets and QD0FF, where the last one is the most important. For further reduction of $\sigma_{y}^{*}$, it is considered to replace the QD0FF magnet

CERN has designed a new quadrupole magnet that meets the QD0FF strength requirements based on permanent material (PM) [32]. In order to carry out the study, the expected multipole components of QF1FF shown in Table IV are assumed for QD0FF. With this assumption, the quadrupole magnets located at high values of the $\beta$ function, namely QD10FF, QF9FF, QF5FF, and QD4FF, plus all the available sextupole magnets are optimized against $\sigma_{y}^{*}$, the minimum $\sigma_{y}^{*}$ obtained is $27 \mathrm{~nm}$, and according to the Shintake definition $\sigma_{y, \text { Shi }}^{*}=25 \mathrm{~nm}$. The impact of the remaining multipole components of the FD and the QEA magnets on the IP beam sizes is still noticeable for the ATF2 ultralow $\beta^{*}$ lattice. From the order-by-order analysis, it is inferred that the octupole component is the most relevant source of vertical beam size increase; see Fig. 4. In addition to that, the obtained beam size when assuming a monochromatic beam is
$\sigma_{y}^{*}\left(\frac{\Delta p}{p}=0\right)=19.5 \mathrm{~nm}$, which indicates that the aberration responsible of the beam size growth is mainly chromatic.

\section{B. Octupole magnets}

A pair of octupole magnets located at a dispersive and nondispersive locations could be used for compensating the chromatic octupole aberration observed in Sec. III A. This solution has been investigated and the results are presented in the following.

Two octupole magnets, namely OCT1FF and OCT2FF, are included as a thin lens in the MAD-X model of ATF2. OCT1FF is placed at the center of SD0FF which is a dispersive location and OCT2FF is inserted at the center of SD4FF which is a nondispersive location, separated by a phase advanced of $180^{\circ}$ from OCT1FF, in order to cancel for the geometric aberrations. With this new FFS configuration, the sextupole and octupole magnets have been optimized against the vertical spot size at the IP. The obtained $\sigma_{y}^{*}$ is $23 \mathrm{~nm}$ when assuming the multipole components of the new design of QD0FF based on PM.

TABLE IV. Comparison between the evaluated tolerance for the ATF2 ultralow $\beta^{*}$ lattice, the measured multipole components of the previously installed QF1FF, the current QF1FF (4Q17) [31], and the expected components of the permanent magnet (PM) [32] at a radius equal to $1 \mathrm{~cm}$ for the sextupole, octupole, decapole, and dodecapole components of QF1FF.

\begin{tabular}{lcccccccr}
\hline \hline \multirow{2}{*}{ QF1FF } & \multicolumn{2}{c}{ Sextupole $10^{-4}$} & \multicolumn{2}{c}{ Octupole $\left[10^{-4}\right]$} & \multicolumn{2}{c}{ Decapole $\left[10^{-4}\right]$} & \multicolumn{2}{c}{ Dodecapole $\left[10^{-4}\right]$} \\
$R=1 \mathrm{~cm}$ & Normal & Skew & Normal & Skew & Normal & Skew & Normal & Skew \\
\hline UL tolerance & $\mathbf{0 . 3 7}$ & $\mathbf{0 . 0 7}$ & $\mathbf{0 . 1 5}$ & $\mathbf{0 . 0 7}$ & $\mathbf{0 . 4 3}$ & $\mathbf{0 . 0 8}$ & $\mathbf{0 . 1 7}$ & $\mathbf{0 . 0 9}$ \\
Replaced & $\mathbf{2 . 7}$ & $\mathbf{0 . 2 8}$ & $\mathbf{0 . 5 7}$ & 0.04 & $\mathbf{1 . 2}$ & $\mathbf{0 . 1 9}$ & $\mathbf{3 . 4}$ & $\mathbf{0 . 7 6}$ \\
4Q17 & 0.28 & $\mathbf{0 . 2}$ & 0.01 & 0.06 & 0.004 & 0.001 & 0.001 & 0.01 \\
PM & -1.8 & $\mathbf{0 . 1 8}$ & -0.28 & 0.02 & -0.5 & -0.08 & 1.2 & -0.26 \\
\hline
\end{tabular}




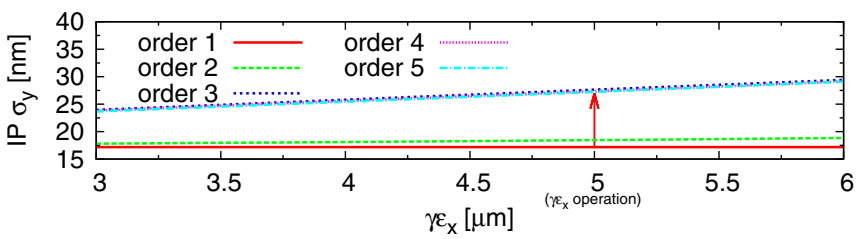

FIG. 4 (color online). $\sigma_{y}^{*}$ evaluated by MAPCLASS as a function of $\gamma \epsilon_{x}$ for the ATF2 ultralow $\beta^{*}$ considering the expected multipole components of the PM magnet for QDOFF. The value of $\gamma \epsilon_{x}$ when operating ATF is $5 \mu \mathrm{m}$.

Yet when considering the multipole components of the present QD0FF magnet a $\sigma_{y}^{*}=24 \mathrm{~nm}$ is obtained. The normalized integrated strength values for the OCT1FF and OCT2FF octupoles for both QD0FF magnets are summarized in Table V, which can be provided by small octupole magnets.

Almost the same spot size is obtained whether the current or the new design of QD0FF are assumed in simulations. Therefore the replacement of QD0FF is not required if the octupoles are installed. Taking this into account inserting a pair of octupole magnets is recommended for testing the feasibility of the challenging $\beta$-optics configuration, if enough free space is available in the FFS of ATF2.

\section{Optics modification}

A different approach to minimize the detrimental impact of the multipole components consists of modifying the optics by increasing $\beta_{x}^{*}$. Doing so, the horizontal beta function is lowered along the FFS. Thus, the impact of all the multipole components present in the ATF2 magnets are reduced as well. Although, increasing $\beta_{x}^{*}$ is not the preferred solution since it is not foreseen in the final focus system design of the future linear colliders, considering ILC as an example, increasing 2.5 times $\beta_{x}^{*}$ enlarges $\sigma_{x}^{*}$ by a factor $\sqrt{2.5}$ which reduces $\mathcal{L}$ by almost $40 \%$. Nevertheless this solution is considered in this study, because it may help to reduce the detrimental effect of the multipole components, enabling the test of the local chromaticity correction scheme of FFS.

By using the matching quadrupoles QM16FF, QM15FF, QM14FF, QM13FF, QM12FF, and QM11FF located at the beginning of the final focus, the $\beta_{x}^{*}$ is increased from 4 to $40 \mathrm{~mm}$. Afterwards the sextupoles are optimized in order to compensate for the beam aberrations and minimize the spot

TABLE V. Integrated strength for the octupole magnets OCT1FF and OCT2FF when considering two different QD0FF magnets, the installed quadrupole and the PM design.

\begin{tabular}{lcc}
\hline \hline QD0FF & OCT1FF $\left[\mathrm{m}^{-3}\right]$ & OCT2FF $\left[\mathrm{m}^{-3}\right]$ \\
\hline Current & -24.16 & 97.90 \\
PM & -13.37 & 98.24 \\
\hline
\end{tabular}

sizes at the IP. This optimization process is done by using the simplex algorithm [33] inserted in MAD-X in combination with the MAPCLASS code responsible to evaluate $\sigma^{*}$.

The new design for the ATF2 nominal lattice with $\beta_{x}^{*}=40 \mathrm{~mm}$ is called ATF2 10Bx1By lattice and features a $\sigma_{y}^{*}=37 \mathrm{~nm}$ which is equal to the one of the ATF2 nominal lattice. The interest of this new design lies in reducing the beam size in the movable tapered beam pipe section used as collimator between QD10A and QD10B quadrupole magnets which reduces the background signal read by the Shintake monitor, easing its operation and also the tuning process. The performance of this new lattice was cross-checked by the ATF2 collaborators [34], using the LUCRETIA code [35], MAD-8 [36], and SAD [37], obtaining similar values of $\sigma_{y}^{*}$. The ATF2 10Bx1By lattice was used for validating the new local chromaticity correction scheme, as reported in [8]. The MAD-X model of the new ATF2 10Bx1By lattice can be found in [38].

Regarding the ATF2 ultralow $\beta^{*}$ lattice, when increasing $\beta_{x}^{*}$ up to $40 \mathrm{~mm}$, the obtained vertical spot size at the IP is $23 \mathrm{~nm}$. Therefore this optics modification solution allows us to effectively minimize the effect of all the measured multipole components. This new lattice design, called ATF2 ultralow $\beta_{y}^{*}$, can be found in [39].

\section{QF1FF MULTIPOLE COMPONENTS OF CLIC AND ILC}

In this section the tolerances for the multipole components of the QF1FF magnet for CLIC $\left(E_{\mathrm{CM}}=3 \mathrm{TeV}\right)$ and ILC $\left(E_{\mathrm{CM}}=0.5 \mathrm{TeV}\right)$ are evaluated. The sextupole, octupole, decapole, and dodecapole tolerances have been calculated at a radius of 1 and $3 \mathrm{~mm}$ for CLIC and ILC, respectively. The calculated tolerances are evaluated at a different radius in order to make a fair comparison due to the difference in beam size at QF1FF, since the CLIC, ILC, and ATF2 operate at different energies. Assuming an errorfree lattice the amount of multipole component is increased until a luminosity loss of $2 \%$ is obtained. The PLACET [40] code has been used to track the beam through the FFS and afterwards the GUINEA-PIG [41] code simulates the interaction of identical $e^{+} e^{-}$beam distributions at the IP; see [42]. The obtained tolerances are shown in Table VI. Comparing the QF1FF tolerances obtained for ATF2 and the LCs, the tolerance of the sextupole components shows an agreement within $10 \%$ between ILC and the ATF2 nominal lattice. Only the skew sextupole component is in agreement within $10 \%$ between CLIC and the ATF2 ultralow $\beta^{*}$ lattice. The disagreement between the tolerances of ATF2 and the LCs is explained by the different criteria used for their calculation. The rms beam size definition used for ATF2 is sensitive to the tails of the bunch while a luminosity calculation is concerned about the core of the beam rather than the tails, as explained in Sec. III. Indeed the higher order components populate the 
TABLE VI. Comparison between the relative multipole components (Rel. Comp.) tolerances of QF1FF magnet at $R=1 \mathrm{~mm}$ for CLIC and $R=3 \mathrm{~mm}$ for ILC. Each tolerance represents a luminosity loss of $2 \%$.

\begin{tabular}{lccccccc}
\hline \hline \multirow{2}{*}{$\begin{array}{l}\text { Rel. Comp. } \\
\text { QF1FF }\end{array}$} & \multicolumn{2}{c}{ Sextupole $\left[10^{-4}\right]$} & \multicolumn{2}{c}{ Octupole $\left[10^{-4}\right]$} & \multicolumn{2}{c}{ Decapole $\left[10^{-4}\right]$} & \multicolumn{2}{c}{ Dodecapole $\left[10^{-4}\right]$} \\
Skew & Normal & Skew & Normal & Skew & Normal & Skew & Normal \\
\hline CLIC $R=1 \mathrm{~mm}$ & 0.4 & 0.2 & 2.5 & 0.5 & 4.0 & 1.7 & 15.0 \\
ILC $R=3 \mathrm{~mm}$ & 1.2 & 0.1 & 1.7 & 0.2 & 3.0 & 0.2 & 4.8 \\
\hline
\end{tabular}

tails of the beam rather than the core, allowing for larger tolerances for the LC. In general tighter tolerances are obtained for ATF2 rather than ILC or CLIC, where CLIC is the project that exhibits the most relaxed tolerances in almost all considered components. This can be explained in terms of the strength of QF1FF. Indeed CLIC runs the weakest QF1FF magnet while ATF2 requires the strongest one, as shown in Table VII.

Although tighter tolerances are observed for ATF2 rather than LCs, measuring the magnetic field components of very long magnets with small apertures, as the ones used in LCs see Table VII, might be a difficult task. It should be noted that satisfying the evaluated tolerances for ILC or CLIC might be equal or even harder as for ATF2 from a magnetic measurement perspective.

\section{CONCLUSIONS}

Analysis of the high order aberrations of the IP beam sizes allows us to determine that the QF1FF magnet installed at ATF2 was mainly responsible for the evaluated beam size growth at the IP for both nominal and ultralow $\beta^{*}$ lattices. By replacing this magnet by the $4 \mathrm{Q} 17$ magnet, which has a better field quality, the expected beam sizes for the ATF2 nominal lattice are recovered. However, the QF1FF replacement does not permit one to reduce the vertical IP beam size beyond $31 \mathrm{~nm}$ for the ATF2 ultralow $\beta^{*}$. For further beam size reduction the replacement of QD0FF by a permanent magnet designed at CERN has been considered, which allows one to squeeze the vertical beam size down to $27 \mathrm{~nm}$. However the insertion of a pair of octupole magnets permits one to bring down $\sigma_{y}^{*}$ to $24 \mathrm{~nm}$, without replacing the QD0FF magnet.

Alternatively, the detrimental impact of multipole components is effectively minimized by increasing the value of $\beta_{x}^{*}$ a factor 10 . Simulations have shown that this solution fully recovers the $\sigma_{y}^{*}$ design for both ATF2 lattices. The new designs are called ATF2 10Bx1By and ultralow $\beta_{y}^{*}$ lattices. Besides minimizing the impact of the multipole

TABLE VII. Comparison between the integrated strength of the QF1FF magnet between ATF2, ILC, and CLIC.

\begin{tabular}{lccc}
\hline \hline & & & $\begin{array}{c}\text { Integrated } \\
\text { strength }\left[\mathrm{m}^{-1}\right]\end{array}$ \\
\hline ATF2 & $\mathrm{L}[\mathrm{m}]$ & $-2]$ & 0.69 \\
ILC & 1.56 & 0.4441 & 0.16 \\
CLIC & 0.08 & 2.0 & 0.13 \\
\hline
\end{tabular}

components they also offer additional features that ease the operation of ATF2, such as low levels of backgrounds and reduction of tuning difficulties.

The ATF2 10Bx1By lattice has been used during the last experimental tuning attempts at ATF2. Significant progress in terms of beam size minimization has occurred since December of 2012. According to the presented simulations the measured multipole components of the ATF2 FFS magnets should not preclude to reach a $\sigma_{y}^{*}=37 \mathrm{~nm}$.

In order to determine the impact of the multipole components of the QF1FF magnet for the future linear colliders, the normal and skew tolerances for CLIC and ILC have been obtained at a radius of 3 and $1 \mathrm{~mm}$, respectively. Even using different radius no clear correspondence can be established between the tolerances of the ATF2 lattices and the tolerances obtained for ILC and CLIC. Tighter tolerances are found for ATF2 rather than the LCs as a result of the strength of QF1FF. Although more relaxed tolerances are found for the LCs projects, achieving the required measurement resolution on long magnets with small apertures might represent a challenge from a magnet measurement point of view.

In conclusion, the study of effect of the multipole component of the ATF2 magnets on the IP beam sizes has allowed one to minimize its detrimental effect by applying the proposed solutions. ATF2 is providing a unique experience to assess the effect of field errors in the final doublet of future colliders with more confidence.

\section{ACKNOWLEDGMENTS}

Work supported by the U.S. Department of Energy under Contract No. DE-AC02-76SF00515.

[1] N. Phinney, N. Toge, and N. Walker, "ILC Reference Design Report Volume 3-Accelerator," Technical Report (2007), http://www.linearcollider.org/cms/?pid=1000437.

[2] B. Barish for the Global Design Effort and S. Yamada for the Research Directorate, The International Linear Collider Technical Design Report (CERN, Geneva, 2013), Technical Report.

[3] ILC home page, http://www.linearcollider.org/ILC/ What-is-the-ILC/The-project.

[4] P. Lebrun, L. Linssen, A. Lucaci-Timoce, D. Schulte, F. Simon, S. Stapnes, N. Toge, H. Weerts, and J. Wells, "The CLIC Programme: Towards a Staged e+e- Linear Collider Exploring the Terascale, CLIC Conceptual Design Report," 
Technical Report (2012), https://edms.cern.ch/document/ 1235960.

[5] R. Tomás, Phys. Rev. ST Accel. Beams 13, 014801 (2010).

[6] CLIC home page, http://clic-study.org.

[7] P. Raimondi and A. Seryi, Phys. Rev. Lett. 86, 3779 (2001).

[8] G. White et al., Phys. Rev. Lett. 112, 014801 (2013).

[9] H. Braun et al., Reports No. CERN-AB-2005-035 and CLIC-Note-636, 2005.

[10] B. Grishanov et al., Reports No. CERN-AB-2006-004 and SLAC-R-796, 2006.

[11] P. Bambade et al., Phys. Rev. ST Accel. Beams 13, 042801 (2010).

[12] P. Bambade et al., Report No. CERN-ATS-2009-092, 2009.

[13] Stanford Linear Accelerator Center, Report No. SLAC-R376, 1991.

[14] V. Balakin et al., Phys. Rev. Lett. 74, 2479 (1995).

[15] V. Alexandrof et al., Particle Accelerators and HighEnergy Accelerators (IEEE, New York, 1996), Vol. 4, pp. 2742-2746.

[16] J. Yan et al., Nucl. Instrum. Methods Phys. Res., Sect. A (to be published).

[17] T. Okugi et al. (unpublished).

[18] T. Shintake, Nucl. Instrum. Methods Phys. Res., Sect. A 311, 453 (1992).

[19] S. Taikan et al., Nucl. Instrum. Methods Phys. Res., Sect. A A616, 1 (2010).

[20] T. Shintake, H. Hayano, A. Hayakawa, Y. Ozaki, M. Ohashi et al., Int. J. Mod. Phys. 2A, 215 (1993).

[21] E. Marin, Ph.D. thesis, Universitat Politecnica de Catalunya (UPC) [Report No. CERN-THESIS-2012-218, 2012 (unpublished)].

[22] R. Tomás, Report No. AB-Note-2006-017 (ABP), 2006.

[23] R. Tomás, Phys. Rev. ST Accel. Beams 9, 5 (2006).

[24] M. Woodley, Repository of the Multipole Component Measurements of the ATF2, http://www.slac.stanford .edu/ mdw/ATF2/v4.4.

[25] M. Masuzawa, "QEA Magnet Measurements at KEK and Comparison with IHEP Results," in the 11th ATF2 Project meeting, 2011, http://ilcagenda.linearcollider.org/ conferenceDisplay.py?confId $=4904$.

[26] S. Bai, P. Bambade, D. Wang, J. Gao, M. Woodley, and M. Masuzawa, Chin. Phys. C 36, 756 (2012).

[27] BE/ABP Accelerator Physics Group of CERN, Mad-X home page, http://frs.home.cern.ch/frs/Xdoc/mad-X.html.

[28] E. Marin et al., in Proceedings of the International Particle Accelerator Conference, Kyoto, Japan (ICR, Kyoto, 2010), pp. 4554-4556, http://accelconf.web.cern.ch/ AccelConf/IPAC10/papers/thpe020.pdf.

[29] T. Henderson, N. Li, J. Osborn, J. Tanabe, D. Yee et al., Conf. Proc. C950501, 1322 (1995).

[30] M. Zisman, R. Yourd, and H. Hsieh, in Proceedings of the Particle Accelerator Conference, Dallas, TX, 1995 (IEEE, New York, 1995), Vol. 1, pp. 533-535.

[31] C. Spencer (private communication).

[32] A. Vorozhtsov, The International Workshop on Future Linear Colliders, Granada, Spain, 2011.

[33] J. A. Nelder and R. Mead, Comput. J. (UK) 7, 308 (1965).

[34] G. White and M. Woodley (private communication).

[35] P. G. Tenenbaum, in Proceedings of the 21st Particle Accelerator Conference, Knoxville, 2005 (IEEE, Piscataway, NJ, 2005).

[36] MAD-8, webpage, http://frs.web.cern.ch/frs/Xdoc/ $\operatorname{mad} 8 w e b / \operatorname{mad} 8 . h t m l$.

[37] Strategic Accelerator Design (SAD), http://acc-physics.kek .jp/SAD/references.html.

[38] G. White and M. Woodley, ATF2 10Bx1By lattice repository, http://code.google.com/p/atf2flightsim/source/ browse/trunk/ATF2/FlightSim/latticeFiles/src.

[39] E. Marin, ATF2 Ultra low betay lattice repository, http:// clicr.web.cern.ch/CLICr/ATF2/New_Multipoles2/ATF2_ Ultra_low_betay.

[40] The tracking code PLACET, https://savannah.cern.ch/ projects/placet/.

[41] GUINEA-PIG++ Summary, https://savannah.cern.ch/ projects/guinea-pig.

[42] Y. Inntjore Levinsen et al., in CLIC Final Focus System (FFS) Multipoles Requirements, ECFA 2013 Hamburg, Germany. 